\title{
Effect of seasonal changes on the innate immunity of wild Pseudois nayaur: potential reason for its endangerment
}

\author{
B. Song ${ }^{2}$, B. $\mathrm{Wu}^{1,2}$ \\ ${ }^{1}$ Key Laboratory of Southwest China Wildlife Resources Conservation, Ministry of Education, Sichuan, PR China \\ ${ }^{2}$ College of Life Science, China West Normal University, Sichuan, PR China
}

[Received: 21 November 2020; Accepted: 11 January 2021; Early publication date: 29 January 2021]

\begin{abstract}
Background: Seasonal climate change may impose stress on mammal's immune system, but this has never been connected to wildlife endangerment reasons. This study focuses on Pseudois nayaur, a rare Chinese sheep facing a severe situation of disappearing.

Materials and methods: Wild Pseudois nayaurs in Sichuan, China were observed in this research by an integrated method including habitat environment, foraging rules, and breeding activities investigation in ecology filed, accompanied with immunity research by peripheral blood, including erythrocyte, neutrophils, natural killer cells, and macrophages immunity by means of erythrocyte rosette, phagocytosis, lactate dehydrogenase release and acid alpha-naphthyl acetate esterase method, respectively.

Results: The results show that Pseudois nayaurs's innate and red blood cell immunity in autumn and winter Pseudois nayaurs and it may be the results of energy reallocation between reproduction and the immune system. In addition, the environment pressure found in Zhu Balong, Sichuan, China including food reduction, human disturbance, low temperature, and severe drought still existed, which may prevent the Pseudois nayaur from normal immunity enhancement before winter.

Conclusions: In conclusion, the innate immunosuppression found in this study especially at their breeding season in autumn and winter may be the ignored cause of wild Pseudois nayaur's endangerment and should be considered in their protection strategy. (Folia Morphol 2022; 81, 1: 107-116)
\end{abstract}

Key words: Pseudois nayaur, ecological immunity, immunosuppression, seasonal change, reproductive coercion

\section{INTRODUCTION}

Pseudois nayaur, a cherished wild species in China under the protection of Chinese government by the law of State Protection of China and the International Union for Conservation of Nature, is in danger of extinction. From 1988 to 2003, their habitat area had decreased about $48,000 \mathrm{~km}^{2}$ in Batang, Sichuan. For the protection of this species, many studied their behavioural traits and living habits. For instance, some mentioned that the group behaviours of them were too

Address for correspondence: Dr. B. Wu, Key Laboratory of Southwest China Wildlife Resources Conservation, Ministry of Education, Shida road 1\#, Nanchong, Sichuan, PR China, tel:+86 13882441768, e-mail: wubangyuan2008@163.com

This article is available in open access under Creative Common Attribution-Non-Commercial-No Derivatives 4.0 International (CC BY-NC-ND 4.0) license, allowing to download articles and share them with others as long as they credit the authors and the publisher, but without permission to change them in any way or use them commercially. 
vulnerable to escape from the predators [19, 28]. Moreover, the skin-based trade was revealed by researches and the Pseudois nayaurs were always easily caught by both human and predators because of the changeless water-drinking places $[20,28]$. As the protection law executed strictly, these predictable factors had been removed. However, the number of the Pseudois nayaurs has not increased as expected; only 500-700 ones were speculated to exist in this area [13]. This indicates that the main reasons for its endangerment are still unknown and unresolved, which asks for more attention to its physiology features, especially for the immune system that determines individuals' death directly.

Animal ecological immunology was defined in the 1990s, covering the study of animal immunity changes under certain environment conditions which were used to wide-ranged science research from physiology to molecular biology $[26,27]$. One of the most important phenomenons to wildlife in the field is the periodic changes in immune system controlled by hormone and innate immunity. It was found that some mammals tend to enhance their innate immunity to get through the living challenges in winter [23], which is mainly correlated to temperature-controlled gene regulation [31]. However, Harshman and Zera [11] studied that a decrease of immunity also existed when the reproduction time is coming up, which means there may be a trade-off relationship between immunity and reproduction [2, 7], but few studies have targeted so far the ones whose reproductive time is between autumn and winter which also asks for energy in immunity enhancement. So, what's the body choice when facing both challenges in reproduction and immunity? Through Pseudois nayaur, the remained questions can be answered, and the relationship between its endangerment and this body choice could also be revealed.

To understand the trade-off relationship between immunity and reproduction in winter-reproduction mammals, and help protect Pseudois nayaur in a more scientific way. The study investigated Pseudois nayaur by the ecological immunology method. Seasonal changes of the immunity were studied by their peripheral blood (PB), mainly reflected by innate and red blood cell (RBC) immunity. Meanwhile, habitat environment, foraging rules were studied to evaluate the environment pressure, and the breeding activities were observed additionally to be combined with immunity research and understand the trade-off choices that the body has made.

\section{MATERIALS AND METHODS}

\section{Experimental animals and ecological research}

Zhu Balong Nature Reserve, Sichuan, China was chosen to be the research area. Thirteen Pseudois nayaurs which were females and healthy were captured first and given a GPS-position collar. Every season (May, August, November, February in the next year), the same individuals were caught again without neutering for the PB (anticoagulated and cooled in ice bag with temperature $0-4^{\circ} \mathrm{C}$ immediately before transfer to a laboratory nearby in $24 \mathrm{~h}$, the total number of samples was 156, 3 replicates $\times 13$ individuals $\times 4$ seasons) from the vena jugularis externa. The manipulating process of animals were supervised by Animal Welfare Committee and Tangjiahe Government under the guidance of the Law of the People's Republic of China on the Protection of Wildlife, 2016, and the blood storage process followed the rules of International Committee standard of haematology, ICSH,1993, using EDTA-2K. Their living habits including foraging rules, reproductive and clustering activities were also trailed through the GPS collar and faeces, before sighted.

\section{Immunological examination}

Routine blood test. The prepared PB transferred to the laboratory was used for routine blood test by the animal haematology analyser (RR-29103302, Mindray); included indexes were white blood cell (WBC), monocytes (Mon), lymphocyte (Lymph), lymphocyte\% (Lymph\%), granulocyte\% (Gran\%), RBC, haematocrit (HCT), mean corpuscular volume (MCV), mean corpuscular haemoglobin $(\mathrm{MCH})$, red blood cell volume distribution width (RDW), mean corpuscular haemoglobin concentration (MCHC), platelet (PLT), platelet count (PCT), mean platelet volume (MPV), haemoglobin (HGB), platelet distribution width (PDW), and monocytes\% (Mon\%). Every data conducted then were recorded and analysed to see the differences between seasons.

Blood smear analysis. The blood smear was made according to the methods used by Ndao et al. [24]. PB slides were made firstly, followed by Wright's-Giemsa solution (G1020, solarbio) staining. Then, the stained slides were pictured through microscope (MF53, Mshot), after which WBC, and RBC were calculated by image-pro plus (HGB can also be reflected by the measurement of light-stained area in blood cell) (Fig. 1).

Detection of the erythrocyte immunity. The erythrocyte rosette rate was measured by the method 


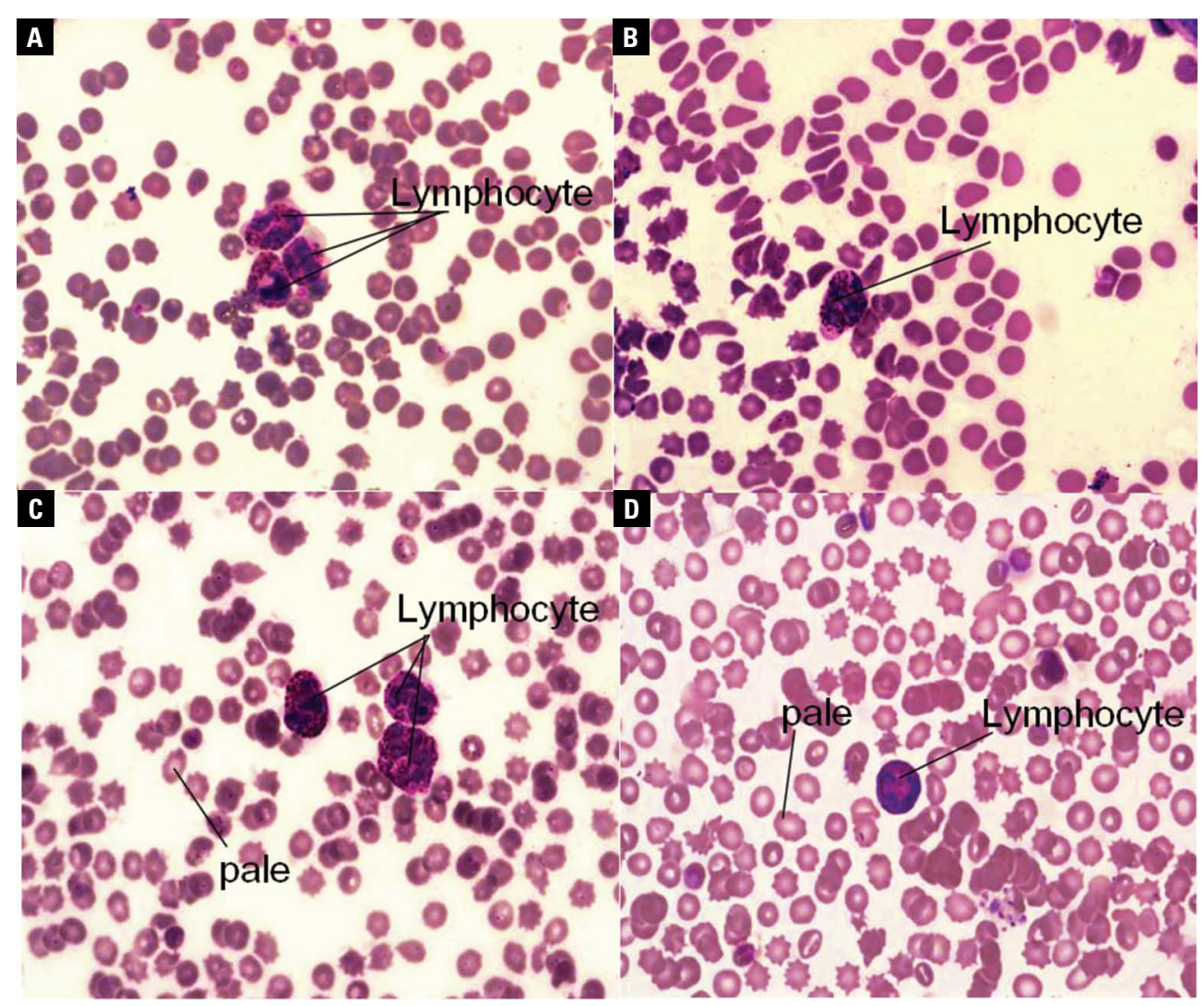

Figure 1. Representative blood smear figures between four seasons. Panels A, B, C, and D are the blood smears in spring, summer, autumn and winter, respectively $(\times 1000$, stained with Wright's-Giemsa staining). The light stained part of red blood cells in autumn and winter was more obvious than that in spring and summer, and the least obvious in summer, indicating that the haemoglobin content in autumn and winter was lower than in spring and summer, while the haemoglobin content in summer was the highest.

which was introduced by Chu et al. [4]. In brief, $4 \mathrm{~mL}$ of yeast solution was taken, and an equal amount of serum was added to make $1 \times 108 / \mathrm{mL}$ concentration of bacterial suspension. Then, the red blood cell suspension was prepared and mixed to make the blood smear. After staining with Wright's-Giemsa, they were detected on a microscopic level. One hundred erythrocytes in each season were calculated. The erythrocyte rosette rate $=$ number of RBCs forming rosette/total number.

Detection of the immune function of neutrophils. Under the condition of sterile operation, 5 drops diluted staphylococcus aureus was added into $1 \mathrm{~mL}$ anticoagulant, mixed and placed in carbon dioxide incubator at $37^{\circ} \mathrm{C}$ for $30 \mathrm{~min}$, and shaken every $10 \mathrm{~min}$. Then, the solution was used to make blood smears, before dried and observed with the microscope (MF53, Mshot). In each season, 100 neutrophils of every individuals were counted, after which the staphylococcus aureus-contained-neutrophils were recorded. The immune function of neutrophils was reflected by this way, and the phagocyte rate $=$ the number of staphylococcus aureus-contained-neutrophils in every 100 neutrophils.

Determination of the NK cell immunological killing function by LDH. Lactate dehydrogenase (LDH) release method was used in the study to the detection of natural kill (NK) cell immunological killing function, as described by Konjević et al. [15]. Briefly, $0.1 \mathrm{~mL}$ of the prepared NK cells of Pseudois nayaur (taken in PB) and $0.1 \mathrm{~mL}$ of the $\mathrm{K} 562$ cells (K562 cells were cultured for $28 \mathrm{~h}$ in logarithmic phase and washed twice with RPMI-1640 medium). Then, they were suspended in 10\% FCS-RPMI-1640 medium at the concentration of $1 \times 105$ cells per $\mathrm{mL}$ and detected by $0.5 \%$ trypan blue staining. Then, among the experimental group, target cell natural release group (0.1 mL target cell $+0.1 \mathrm{~mL} \mathrm{10 \%} \mathrm{FCS-RPMI-1640}$ medium) and maximum release group $(0.1 \mathrm{~mL}$ target cell $+0.1 \mathrm{~mL} 1 \% \mathrm{NP} 40$ solution) were set up. After adding $L D H$ substrate liquid for $15 \mathrm{~min}$, the value $A$ was measured by Enzyme Linked Monitor (ELx800, Bio-Tek, USA) under $570 \mathrm{~nm}$. NK cell activity \% = [(value $A$ of the experimental group - value $A$ of 
natural release control group)/(value $\mathrm{A}$ of maximum - release control group)] $\times 100 \%$.

Detection of the phagocytosis of macrophages. Each blood smear slide of four seasons was prepared in the same way as mentioned above. After that, they were fixed with the solution of alpha-naphthalene acetic acid ( $\alpha$-NAE) for $15 \mathrm{~min}$, then washed with distilled water for $5 \mathrm{~min}$ and dried under $25^{\circ} \mathrm{C}$. Then, the $\alpha$-NAE incubation solution was added, before put the smears into the humid cabinet at $37^{\circ} \mathrm{C}$ (avoiding light to incubate for $1 \mathrm{~h}$ ). To the next, after cleaning with water, the solution of methyl green dye was added to the droplet and then restained with water, after which the positive rate of macrophages was calculated under the microscope (MF53, Mshot) by the image-pro plus. (All reagents were purchased from the $\alpha$-NAE assay kit, CAT\#G2390, Solarbio, Japan).

\section{Statistical analysis}

The data collected from PB experiments mentioned above were analysed with t-test, one-way ANOVA. The data passed the homogeneity test for variance went through the Least-Significant Difference (LSD) test, and others were compared by Tamhane's $T 2$ test to keep the findings about the impact of seasons on Pseudois nayaurs' immunity in accuracy. For the calculation process, it was done by SPSS20, and the outcomes were displayed as means \pm standard deviation $(x \pm S D), p$-value, $d f, F$, and MS figures.

\section{RESULTS}

\section{Ecological results}

Research area. The Pseudois nayaurs lived in Zhubalong preserve with an altitude between 2,600 and $3,500 \mathrm{~m}$. This area is a mountain valley landform and locates in the south-eastern edge of the Qinghai-Tibet plateau. It was found that a large number of Pseudois nayaurs prefer staying at the places with cliff to be able to escape from their natural enemies. In addition, the climate here was obviously divided into dry season (from October to the next April) and rainy season (from May to September) every year, which makes winter harder to get through. To cope with the unfriendly climate, the concave hillside was used by them, which was both helpful for the cover under the rain and the warming up under the sunshine. For instance, in summer, the Pseudois nayaur would move their sleeping place to a cool place to avoid the high temperature, strong sunshine, or rain. In winter, they tended to choose steep slopes under the sun as resting points.
Foraging rules. In this research, we observed that the foraging time of the Pseudois nayaurs was mainly on the morning and evening. Meadows, thickets, rocky escarpments with sparse grasses, and tall oak forests were the usual foraging sites. It was found that the main vegetation coverage was spiny roses and annual herbs, which accorded with the characteristics of vegetation coverage in dry-hot valley climate. However, the amount of the available food for Pseudois nayaurs was not in lacking. Dry environments and low temperatures limited the growth of vegetation, besides, grazing activities led by human were also found, which imposed threats to their living conditions, especially in autumn and winter.

Breeding activities. In the aspect of breeding activities, we found that the time was mainly in November, which is at the boundary time of autumn and winter. Pregnant females give birth to offspring in May of the following year after about 6 months of gestation. Due to the harsh winter environment during pregnancy and the influence of natural enemies on the offspring, both the pregnancy success rate and the survival rate of the offspring were low, we speculated that this was an important reason why the species is in the danger of extinction and was ignored by most scholars.

Cluster activities. For group behaviours, the cluster types were observed in mixed group, separated female group and male group. The number of mixed groups was the largest, mostly 20 to 30 individuals per group. This might be the strategy of them to survival from predators and harsh climates, while it became harder and harder to keep that group number.

\section{Immunological detection}

Blood routine. The results of blood routine are shown in Tables 1-3. For the immune system related cells figures in Table 1, there was no significant difference between seasons for WBC and Mon ( $p>0.05$ ). However, the number of Lymphs decreased rapidly in autumn, compared with the figures in spring ( $p<0.01)$ and summer $(p<0.05)$, before climbed to the highest in winter $(p<0.01$, compared with the figure in autumn). For the figure of Gran, it was seen a decrease in autumn compared with the figure in winter $(p<0.05)$.

To RBCs related figures shown in Table 2, everyone except for RDW and MCV was significantly low in autumn and increased at different levels in winter. It should be noticed that $\mathrm{MCH}, \mathrm{MCHC}$, and $\mathrm{HGB}$ had apparently 
Table 1. Immunity related cells' blood routine results

\begin{tabular}{lccccccc}
\hline Project & Spring & Summer & Autumn & Winter & df & F & MS \\
\hline WBC $\left[10^{9} / \mathrm{L}\right]$ & $8.62 \pm 0.76$ & $7.69 \pm 0.22$ & $7.28 \pm 3.74$ & $8.80 \pm 0.22$ & 155 & 0.146 & 1.604 \\
Monocytes $\left[10^{9} / \mathrm{L}\right]$ & $2.33 \pm 0.28$ & $1.83 \pm 0.15$ & $0.59 \pm 0.55$ & $2.50 \pm 0.1$ & 155 & 7.109 & 2.231 \\
Lymphocyte $\left[10^{9} / \mathrm{L}\right]$ & $8.17 \pm 1.53^{\mathrm{c}}$ & $7.13 \pm 0.46^{\mathrm{c}}$ & $2.57 \pm 1.21^{\mathrm{AbD}}$ & $9.27 \pm 0.40^{\mathrm{c}}$ & 155 & 8.243 & 25.983 \\
Granulocyte $\left[10^{\circ} / \mathrm{L}\right]$ & $7.57 \pm 0.58$ & $6.84 \pm 0.16$ & $6.47 \pm 0.22^{\mathrm{d}}$ & $7.62 \pm 0.23^{\mathrm{c}}$ & 155 & 2.717 & 0.955 \\
\hline
\end{tabular}

WBC - white blood cell; Data are expressed as mean and standard deviation (sample number $=156$ ); $a, b, c, d$ means different from those data in spring, summer, autumn and winter respectively. Capitalisation means significant difference $p<0.01$, while lowercase represent extremely significant $p<0.05$. Overall, severe immunosuppression occurred in autumn, followed by winter, but in a resistant state.

Table 2. Red blood cells related blood routine results

\begin{tabular}{|c|c|c|c|c|c|c|c|}
\hline Project & Spring & Summer & Autumn & Winter & df & $\mathbf{F}$ & MS \\
\hline $\mathrm{RBC}\left[10^{12} / \mathrm{L}\right]$ & $5.86 \pm 0.49$ & $5.94 \pm 0.07$ & $3.30 \pm 1.62^{d}$ & $6.34 \pm 0.36^{c}$ & 155 & 2.580 & 5.807 \\
\hline НСТ [\%] & $35.63 \pm 2.90$ & $37.40 \pm 1.04$ & $21.23 \pm 10.53^{c}$ & $39.87 \pm 1.68^{d}$ & 155 & 2.282 & 210.762 \\
\hline $\operatorname{MCV}[f \mathrm{~L}]$ & $60.97 \pm 0.62^{\mathrm{a}}$ & $62.80 \pm 1.23$ & $65.53 \pm 1.81^{\mathrm{c}}$ & $63.00 \pm 0.96$ & 155 & 2.266 & 10.570 \\
\hline $\mathrm{MCH}[\mathrm{pg}]$ & $50.27 \pm 0.96^{c}$ & $50.63 \pm 0.52^{c d}$ & $24.00 \pm 0.17^{A B D}$ & $48.33 \pm 0.37^{\mathrm{abc}}$ & 155 & 489.131 & 500.136 \\
\hline RDW [\%] & $13.23 \pm 0.55$ & $13.47 \pm 0.98$ & $16.17 \pm 1.84$ & $13.47 \pm 1.05$ & 155 & 1.346 & 5.823 \\
\hline MCHC $[g / L]$ & $826.00 \pm 7.55^{c d}$ & $806.67 \pm 25.33^{C}$ & $373.33 \pm 4.91^{\mathrm{ABD}}$ & $768.33 \pm 5.46^{a C}$ & 155 & 245.296 & 138469.639 \\
\hline $\mathrm{HGB}[\mathrm{g} / \mathrm{L}]$ & $294.33 \pm 22.67^{C}$ & $302.67 \pm 0.67^{c}$ & $117.33 \pm 6.06^{\mathrm{ABD}}$ & $306.67 \pm 15.01^{c}$ & 155 & 43.668 & 25440.528 \\
\hline
\end{tabular}

RBC — red blood cell; HCT — haematocrit; MCV — mean corpuscular volume; $\mathrm{MCH}$ — mean corpuscular haemoglobin; RDW — red blood cell volume distribution width; MCHC — mean corpuscular haemoglobin concentration; HGB - haemoglobin; Data are expressed as mean and standard deviation (sample number $=156$ ); $a, b, c, d$ means different from those data in spring, summer, autumn and winter respectively. Capitalisation means significant difference, $p<0.01$, while lowercase represent extremely significant $p<0.05$. Overall, severe immunosuppression occurred in autumn, followed by winter, but in a resistant state.

Table 3. Platelet related blood routine results

\begin{tabular}{|c|c|c|c|c|c|c|c|}
\hline Project & Spring & Summer & Autumn & Winter & df & $\mathbf{F}$ & MS \\
\hline PLT [\%] & $524.33 \pm 152.4^{\text {bd }}$ & $1016.33 \pm 215.48^{\mathrm{ac}}$ & $396.67 \pm 117.32^{\mathrm{bD}}$ & $1132.00 \pm 56.08^{a C}$ & 155 & 6.028 & 391425.556 \\
\hline РCT [\%] & $0.25 \pm 0.07^{\mathrm{bd}}$ & $0.52 \pm 0.09^{a}$ & $0.29 \pm 0.09^{d}$ & $0.57 \pm 0.03^{\mathrm{ac}}$ & 155 & 4.746 & 0.078 \\
\hline MPV [fl] & $4.87 \pm 0.23$ & $4.83 \pm 0.09$ & $5.37 \pm 0.37$ & $5.00 \pm 0.10$ & 155 & 1.136 & 0.179 \\
\hline PDW [fL] & $16.33 \pm 0.33^{c}$ & $15.67 \pm 0.33^{C}$ & $12.63 \pm 0.59^{A B D}$ & $16.00 \pm 0.00^{c}$ & 155 & 20.286 & 8.723 \\
\hline
\end{tabular}

PLT — platelet; PCT — platelet count; MPV — mean platelet volume; PDW — platelet distribution width; Data are expressed as mean and standard deviation (sample number = 156); $a, b, c, d$ means different from those data in spring, summer, autumn and winter respectively. Capitalisation means significant difference $p<0.01$, while lowercase represent extremely significant $p<0.05$. Overall, severe immunosuppression occurred in autumn, followed by winter, but in a resistant state.

significant decreases $(p<0.01)$ in autumn since spring, and then, recovered with an inadequate level in winter.

As for PLT related indexes, the figure for PLT was at the lowest level in autumn, compared to the highest one in winter $(p<0.01)$. In addition, the trend for the change of PDW was dramatic; it decreased considerably from spring to autumn ( $p<0.01)$, which reached the bottom, and increased steadily at winter. For MPV, no evidence had shown the changes between seasons ( $p>0.05)$, and there were both relatively low figures found in spring and autumn for PCT.

Overall, whether indexes in RBCs, PLT, or immune related cells, wide-range decreases were found. Meanwhile, the recovery seemed to happen in winter but in a sign of weakness.
Blood smear analysis. The light stained part of RBCs in autumn and winter was more obvious than that in spring and summer (least obvious), indicating that the HGB contents in autumn and winter were lower in spring and summer, shown in the Table 4.

Erythrocyte immunity. The results are shown in Figure 2. The rosette rate of RBCs decreased significantly in winter $(p<0.05)$ when compared with the figures in spring, summer, and autumn. The results showed that the immune function of erythrocytes was inhibited in winter.

Immune function of neutrophils. As shown in Figures 3 and 4, seasonal changes had a significant impact on the number of neutrophils ( $p<0.01$ ). The phagocytosis rate of neutrophils in summer was 
Table 4. Blood smear cell count analysis

\begin{tabular}{lccccccc}
\hline Project & Spring & Summer & Autumn & Winter & df & F & MS \\
\hline WBC $\left[10^{9} / L\right]$ & $8.58 \pm 0.76$ & $7.51 \pm 0.22$ & $7.27 \pm 3.74$ & $8.86 \pm 0.22$ & 155 & 0.165 & 1.625 \\
RBC $\left[10^{12} / L\right]$ & $5.71 \pm 0.49$ & $5.91 \pm 0.07$ & $3.46 \pm 1.62^{\mathrm{d}}$ & $6.82 \pm 0.36^{\mathrm{c}}$ & 155 & 2.731 & 5.994 \\
\hline
\end{tabular}

RBC — red blood cell; WBC — white blood cell; Data are expressed as mean and standard deviation (sample number $=156$ ); $a, b, c, d$ means different from those data in spring, summer, autumn and winter, respectively. Capitalization means significant difference $p<0.01$, while lowercase represent extremely significant $p<0.05$. Table 4 shows that red blood cells decreased significantly in autumn which indicated the reproduction stress in that time. And increased in winter, which was thought to be relevant to the compensatory strategies.

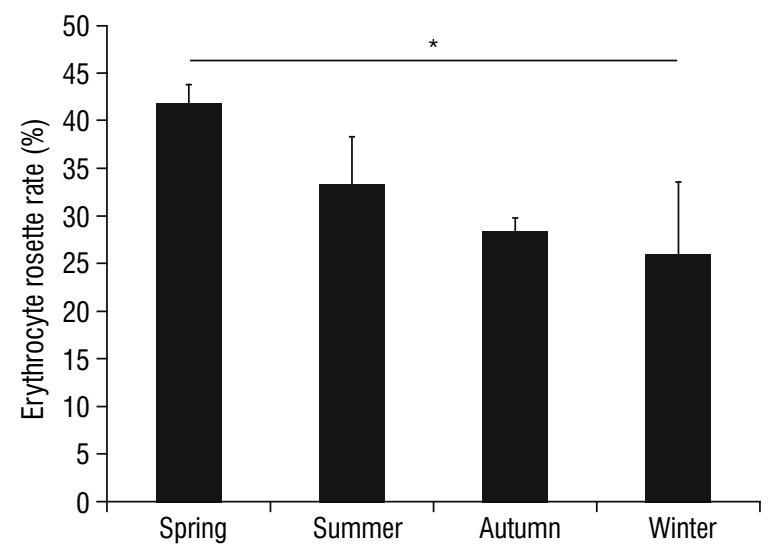

Figure 2. Statistical chart of the seasonal changes of the erythrocyte wreath rate. ${ }^{*}$ In the figure represents a significant difference between the two groups $(p<0.05)$. The comparison between each group and the winter group was conducted before pairwise comparison. The rosette rate of red blood cells significantly decreased in winter $(p<0.05)$ when compared with the spring, was low summer and autumn. The results showed that the immune function of erythrocytes was inhibited in winter.

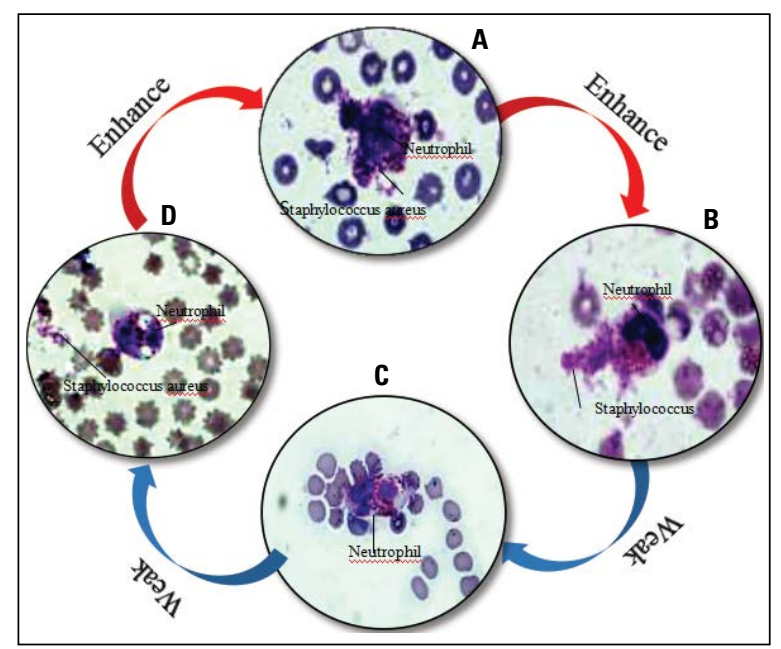

Figure 3. Seasonal changes of staphylococcus aureus phagocytised by neutrophils. Panels A, B, C, and D are the blood smears in spring, summer, autumn and winter, respectively $(\times 1000$, stained with Wright's-Giemsa staining). Phagocytosis rate of neutrophils in summer was significantly higher than in winter $(p<0.01)$, the indexes in summer and autumn were higher than those in spring and winter, respectively $(p<0.05)$.

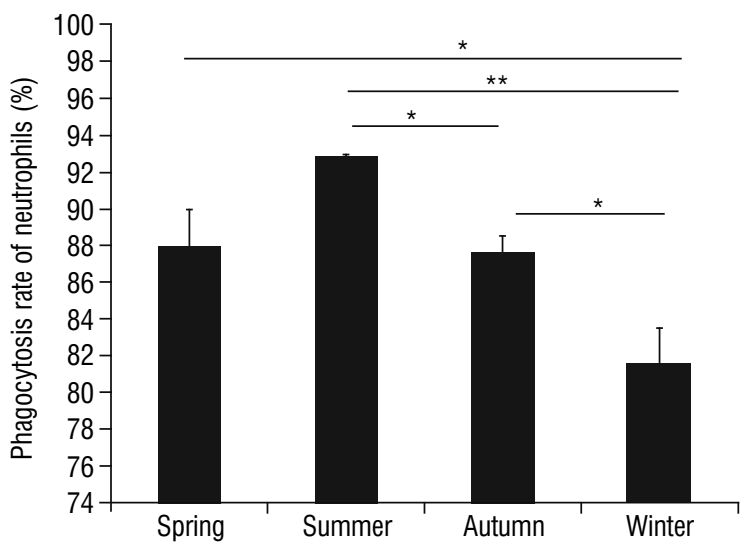

Figure 4. The statistical diagram of the seasonal changes of staphylococcus aureus phagocytised by neutrophils. * In the figure represents a significant difference between the two groups $(p<0.05)$, and ${ }^{* *}$ represents a very significant difference $(p<0.01)$. The figure suggests that the function of neutrophils was affected by the seasonal changes. It is better in summer than in spring and autumn, while the suppression was found in winter.

significantly higher than that in winter $(p<0.01)$, and the indexes in summer and autumn were higher than those in spring and winter, respectively $(p<0.05)$. The results showed that the bactericidal function of neutrophils was inhibited in winter, and was the strongest in summer, followed by spring and autumn.

Phagocytosis of macrophages. As shown in Figure 5, there were significant differences between seasons (except for them between autumn and winter) of the figures $(p<0.01)$, and the figures were the lowest in autumn and winter. The results showed that the function of macrophages was inhibited in autumn and winter, while it was relatively stronger in spring and especially in summer.

NK cell immunological killing function. As shown in Figure 6, seasonal changes had imposed great influence of the function for NK cells, they could only played the weakest role in autumn and winter, compared with that in spring and summer. 


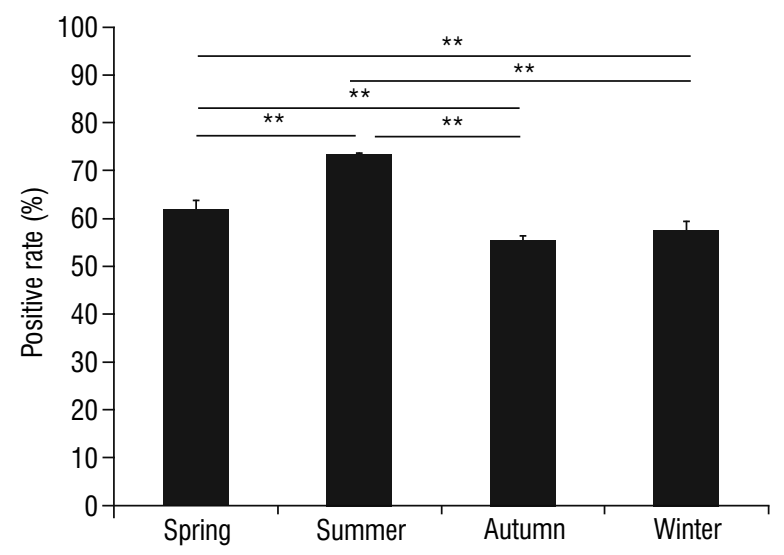

Figure 5. Seasonal variation of macrophage positive rate. ${ }^{* *}$ In the figure represents a very significant difference $(p<0.01)$. It can be concluded in the figure that the macrophage positive rate was also in change with the period of seasons, but it was in the autumn that the rate declined more significantly instead of winter, which was speculated to be relevant to the reproduction matter.

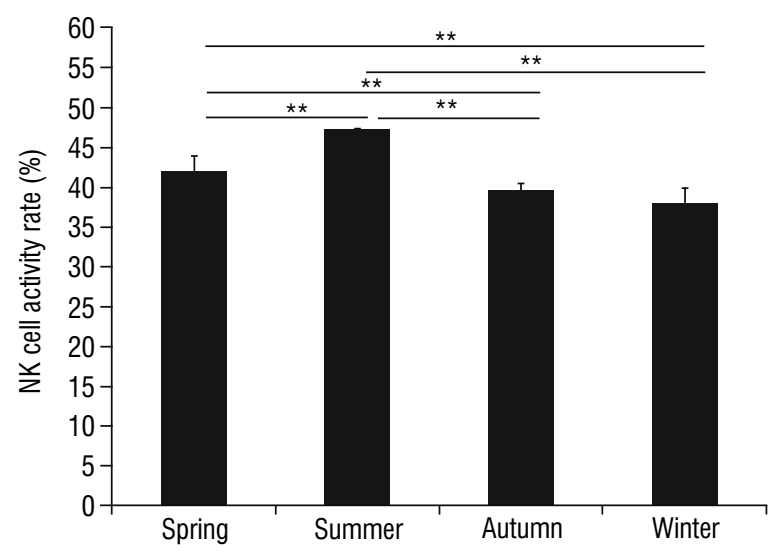

Figure 6. Statistical diagram of the activity rate of natural kill (NK) cells in four seasons. ${ }^{* *}$ In the figure represents a very significant difference $(p<0.01)$. NK cell activity rate was shown in an increase in summer which was consistent to above indexes, and the rate in spring and autumn, was familiar but declined in winter.

\section{DISCUSSION}

\section{Challenging living conditions}

In this research, four aspects of living conditions were studied, including habitat environment, foraging rules, breeding and cluster activities. Firstly, the places that Pseudois nayaur lives in locate in the south-eastern edge of the Qinghai-Tibet plateau, where mountain valley and cliffs are usually to be seen. It was found that they could take good advantages of the landform to escape from predators, and achieve the possibility of keeping safe from harmful sunshine and pour rain. However, step down to the bottom of the valley for water was unpreventable when the dry season (autumn and winter) was coming, which had been become the most risky movement of Pseudois nayaurs because of the awaiting hunter traps and natural enemies. What's more, although the food available for the Pseudois nayaur was relatively enough in spring and summer, dry season had again made the vegetation in degradation especially for the higher but safer place. When facing such pressure, clusters got its own strategy, mainly expressed by acting together. As a result, more strong and healthy individuals would survive, but apparently, most females whose pregnancy period of time were from November to May are not among the group, which was proved by the low survival rate of cubs and the mothers found by Liu et al. [19].

\section{Trade-off between immune and reproduction of Pseudois nayaur}

In addition to the external challenges, the energy cost of reproduction and immunity in autumn and winter also should be noticed. It has been found that, body usually make choices between reproduction and immunity, because the energy gained is limited. For most of the time, the immunity would be the suppressed one $[6,8,14]$. This hypothesis can be the reason why Pseudois nayaur's immunity was found in a sigh of weakness in autumn. Among them, PLT and PCT, shown in Table 2, were at a low condition. Kuter and Rosenberg [18] did a correlation analysis about the reason why PLT was low, the factors leading to the destruction of PLT in the experiment, such as diseases of the Pseudois nayaur, were excluded. We believed that this phenomenon may be related to the reduction of megakaryocytic cells and growth factors in bone marrow, resulting in the weakened proliferation and differentiation of PLT, thus the function of wound healing was reduced. Then, MCH, MCHC, and HGB indexes showed seasonal suppression which indicated that malnutrition was exist and also suggested that the blood's ability to transport oxygen decreased obviously in autumn, and in this period Pseudois nayaur may face to the risk of anaemia disorders $[3,9]$. These findings also provided evidence of food shortages and the effects of overgrazing in the results. In terms of innate immune function, NK cells was inhibited, indicating the rising risk of infection, transformation or stress and decreased target cells kill function [30], even much 


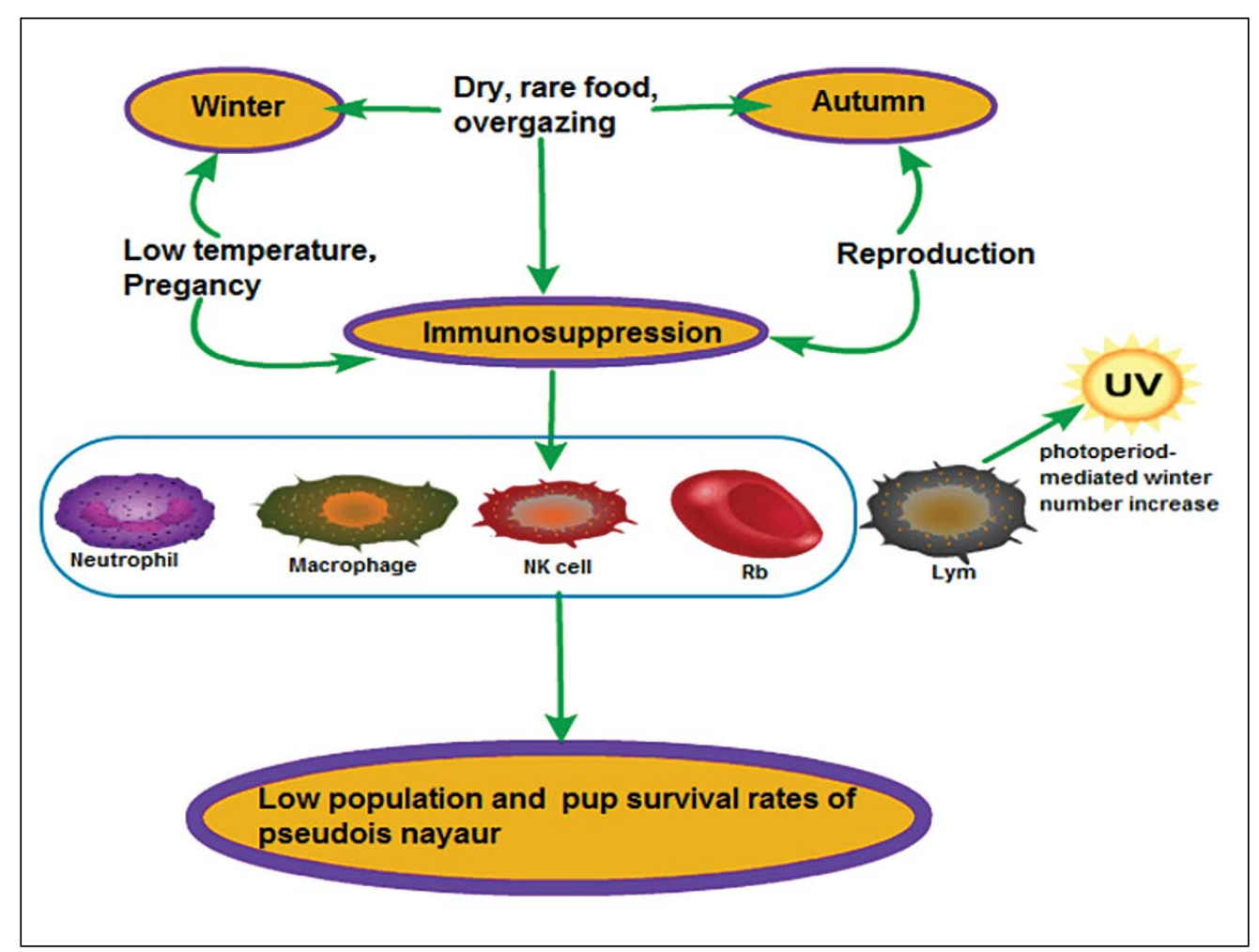

Figure 7. Eco-immune pathway of endangered of Pseudois nayaur. The immunosuppression may be due to the seasonal changes and reproduction hobby. The harsh climates and human activities causes the immunosuppression against the evolutionary strategies that $P$ seudois nayaur owns to enhance the immunity, and it may finally result in low survival rates.

higher probability of getting cancer [1]. Moreover, the macrophages and neutrophils functions were inhibited, which meant the second line of immune defence decreased and bacterial resistance was declined [10, 22]. In addition, erythrocyte rosette rate's suppression in results was also a good proof of that, because the erythrocyte plays an important part in immune complex clearing, tumour, and innate immune $[5,21]$.

\section{Winter immunity recovery}

In winter, the immunity suppression was also found but in a lower level. However, the food and water pressure were bigger than that in autumn, and the effects of low temperature were also beginning to emerge. So, the results were thought to be an evidence for the winter immunoenhancement hypothesis.

Through ecological investigation, it was found that cliffs and concave hillside were used by Pseudois nayaur gaining appropriate sunshine, and it is believed that many small mammals have evolved a set of life-cycle strategies that can increase their immune function by using seasonal variation signals such as sunshine trait (length and strength) to improve their survival in winter $[25,29]$. In this study, WBC was found the highest in winter and the upward trend was also obvious for the recovery of immunity in many other immunology findings, which are complied with similar phenomenon found previously in root voles. The rising number of WBC and other indexes would make them survive better in winter $[16,17]$. The rise of MCV in autumn might suggest the mechanism of resistance to the reduction of $\mathrm{MCH}$ and MCHC. For the further evidence to the phenomenon, studies in molecular level are needed. Although, the phenomenon of resistant immunosuppression was found, the future of the Pseudois nayaur was not encouraging. After the trade-off choice has been made in autumn to enhance the ability to reproduce at the expense of immunity, winter resistance seemed very important for their survival, and therefore, more efficient steps needs to be taken at this very season. The key point of its protection then turns to environment again, but in more specific to winter. We need to stop the agricultural overgrazing [12] and other human interference or wild Pseudois nayaur's extinction would be destination. 


\section{CONCLUSIONS}

As a conclusion, we suggest that the habitat pressure of this species keeps increasing, and it is also found that these species have a large-scale immunosuppression when it is close to the special autumn breeding period in winter (Table 1), which confirms the trade-off between immunity and reproduction. At the same time, due to the challenge of winter climate, the immunity has not recovered quickly though there is a phenomenon of winter immunoenhancement. As shown in results, the survival of female Pseudois nayaur during pregnancy was difficult. Therefore, we conclude that the immune suppression of Pseudois nayaur is more threatening than other species. They are facing an immunosuppression for up to 6 months which is also the time for reproduction in population (Fig. 7).

\section{Acknowledgements}

Authors would like to thank the co-workers of China West Normal University and Zhu Balong Nature Reserve for their assistance in performing the experiment and the analysis. The study was supported by the programme for Education Department of Sichuan (project no. 17ZB0425). And, it was full-time supervised by Animal Welfare and Protection Committee of China West Normal University, the animals used in this studied were anesthetised firstly before the blood taking, and general health was also checked before and after the experiment to ensure their ability of living was not hurt and the accuracy of this research.

\section{Conflict of interest: None declared}

\section{REFERENCES}

1. Albertsson PA, Basse PH, Hokland M, et al. NK cells and the tumour microenvironment: implications for NK-cell function and anti-tumour activity. Trends Immunol. 2003; 24(11): 603-609, doi: 10.1016/j.it.2003.09.007, indexed in Pubmed: 14596885.

2. Amdam GV, Aase AL, Seehuus SC, et al. Social reversal of immunosenescence in honey bee workers. Exp Gerontol. 2005; 40(12): 939-947, doi: 10.1016/j.exger.2005.08.004, indexed in Pubmed: 16169181.

3. Cartwright GE, Lee GR. The anaemia of chronic disorders. Br J Haematol. 1971; 21(2): 147-152, doi: 10.1111/j.13652141.1971.tb03424.x, indexed in Pubmed: 4934605.

4. Chu Y, Haigh T, Nash GB. Rheological analysis of the formation of rosettes by red blood cells parasitized by Plasmodium falciparum. Br J Haematol. 1997; 99(4): 773-783, doi: 10.1046/j.1365-2141.1997.4643268.x, indexed in Pubmed: 9432021.
5. Cornacoff JB, Hebert LA, Smead WL, et al. Primate erythrocyte-immune complex-clearing mechanism. J Clin Invest. 1983; 71(2): 236-247, doi: 10.1172/jci110764, indexed in Pubmed: 6822663.

6. Deerenberg C, Arpanius V, Daan S, et al. Reproductive effort decreases antibody responsiveness. P Roy Soc B-Biol Sci. 1997; 264(1384): 1021-1029, doi: 10.1098/ rspb.1997.0141.

7. Fedorka KM, Zuk M, Mousseau TA. Immune suppression and the cost of reproduction in the ground cricket, Allonemobius socius. Evolution. 2004; 58(11): 2478-2485, doi: 10.1111/j.0014-3820.2004.tb00877.x, indexed in Pubmed: 15612291.

8. French SS, DeNardo DF, Moore MC. Trade-offs between the reproductive and immune systems: facultative responses to resources or obligate responses to reproduction? Am Nat. 2007; 170(1): 79-89, doi: 10.1086/518569, indexed in Pubmed: 17853993.

9. Fuchs D, Hausen A, Reibnegger G, et al. Immune activation and the anaemia associated with chronic inflammatory disorders. Eur J Haematol. 1991; 46(2): 65-70, doi: 10.1111/j.1600-0609.1991.tb00524.x, indexed in Pubmed: 1899833.

10. Gordon S, Martinez FO. Alternative activation of macrophages: mechanism and functions. Immunity. 2010; 32(5): 593-604, doi: 10.1016/j.immuni.2010.05.007, indexed in Pubmed: 20510870.

11. Harshman LG, Zera AJ. The cost of reproduction: the devil in the details. Trends Ecol Evol. 2007; 22(2): 80-86, doi: 10.1016/j.tree.2006.10.008, indexed in Pubmed: 17056152.

12. Homewood K, Rodgers WA. Pastoralism, conservation and the overgrazing controversy; In Conservation in Africa: people, policies and practice. (eds) Anderson D and Grove R. Cambridge University Press, London 1987: 111-128.

13. Hu JC. Dwarf rock sheep in urgent need of protection. China Nat. 1997; 1: 34-35.

14. Knowles S, Nakagawa S, Sheldon B. Elevated reproductive effort increases blood parasitaemia and decreases immune function in birds: a meta-regression approach. Funct Ecol. 2009; 23(2): 405-415, doi: 10.1111/j.13652435.2008.01507.x.

15. Konjević G, Jurišić V, Spužić I. Corrections to the original lactate dehydrogenase (LDH) release assay for the evaluation of NK cell cytotoxicity. J Immunol Methods. 1997; 200(1-2): 199-201, doi: 10.1016/s00221759(96)00194-9.

16. Książek A, Zub K, Szafrańska PA, et al. The nexus of hair corticosterone level, immunocompetence, metabolic rates and overwinter survival in the root vole, Microtus oeconomus. Gen Comp Endocrinol. 2017; 250: 46-53, doi: 10.1016/j.ygcen.2017.05.021, indexed in Pubmed: 28577898.

17. Książek A, Zub K, Szafrańska PA, et al. Immunocompetence and high metabolic rates enhance overwinter survival in the root vole, Microtus oeconomus. Biol Lett. 2014; 10(12): 20140684, doi: 10.1098/rsbl.2014.0684, indexed in Pubmed: 25519753.

18. Kuter DJ, Rosenberg RD. Appearance of a megakaryocyte growth-promoting activity, megapoietin, during acute 
thrombocytopenia in the rabbit. Blood. 1994; 84(5): 1464-1472, indexed in Pubmed: 8068941.

19. Liu G, Zhou C, Yang Z, et al. Diurnal activity rhythm and time budgets of the Dwarf Blue Sheep (Pseus schaeferi) in Zhubalong Nature Reserve. Acta Ecol Sinica. 2011; 31(4): 00197-0981, doi: 10.3724/SP.J.1011.2011.00197.

20. Long S, Zhou CQ, Wang WK, et al. Behavior coding and ethogram of the pseudois nayaur. Acta Ecol Sinica. 2008; 28(11): 5632-5640.

21. Lutz HU. nnate immune and non-immune mediators of erythrocyte clearance. Cell Mol Biol. 2004; 50(2): 107-116, doi: 10.1379/1466-1268(2004)009<0088: $\mathrm{AOHIVI}>2.0 . \mathrm{CO} ; 2$.

22. MacMicking J, Xie QW, Nathan C. Nitric oxide and macrophage function. Annu Rev Immunol. 1997; 15: 323-350, doi: 10.1146/annurev.immunol.15.1.323, indexed in Pubmed: 9143691.

23. Martin LB, Weil ZM, Nelson RJ. Seasonal changes in vertebrate immune activity: mediation by physiological tradeoffs. Philos Trans R Soc Lond B Biol Sci. 2008; 363(1490): 321-339, doi: 10.1098/rstb.2007.2142, indexed in Pubmed: 17638690 .

24. Ndao M, Bandyayera E, Kokoskin E, et al. Comparison of blood smear, antigen detection, and nested-PCR methods for screening refugees from regions where malaria is endemic after a malaria outbreak in Quebec, Canada. J Clin Microbiol. 2004; 42(6): 2694-2700, doi: 10.1128/ JCM.42.6.2694-2700.2004, indexed in Pubmed: 15184454.
25. Nelson R, Demas G. Seasonal changes in immune function. Quarterly Rev Biol. 1996; 71(4): 511-548, doi: 10.1086/419555.

26. Rolff J, Siva-Jothy MT. Invertebrate ecological immunology. Science. 2003; 301(5632): 472-475, doi: 10.1126/ science.1080623, indexed in Pubmed: 12881560.

27. Sheldon BC, Verhulst S. Ecological immunology: costly parasite defences and trade-offs in evolutionary ecology. Trends Ecol Evol. 1996; 11(8): 317-321, doi: 10.1016/0169-5347(96)10039-2, indexed in Pubmed: 21237861.

28. Shen DJ, Wang Y, Zeren J, et al. Primary study on behavior of dwarf blue sheep (pseus schaeferi). Sichuan J Zool; 2007: 26-4 doi org/10 1016/0169. 2007; 2007(26-4), doi: 10.1016/0169-5347(96)10039-2.

29. Sinclair JA, Lochmiller RL. The winter immunoenhancement hypothesis: associations among immunity, density, and survival in prairie vole (Microtus ochrogaster) populations. Can J Zool. 2000; 78(2): 254-264, doi: 10.1139/ z99-203.

30. Sun JC, Lanier LL. NK cell development, homeostasis and function: parallels with $\mathrm{CD} 8^{+} \mathrm{T}$ cells. Nat Rev Immunol. 2011; 11(10): 645-657, doi: 10.1038/nri3044, indexed in Pubmed: 21869816.

31. Xaquin CD, Marina E, Ricardo CF, et al. Widespread seasonal gene expression reveals annual differences in human immunity and physiology. Nat Commun. 2015; 6(1), doi: 10.1038/ncomms8000. 